\title{
Enhancing ECN's abilities to address inventive strategies using OTSM-TRIZ
}

\section{Nikolai Khomenko, Roland De Guio* and Denis Cavallucci}

INSA de Strasbourg, 24, bd de la victoire, F-67084 Strasbourg, France

E-mail: Nikolai.khomenko@insa-strasbourg.fr

E-mail: roland.deguio@insa-strasbourg.fr

E-mail: denis.cavallucci@insa-strasbourg.fr

${ }^{*}$ Corresponding author

\begin{abstract}
This paper analyses and discusses from various points of views to what extent Theory of Inventive Problem Solving (TRIZ) and its generalisation (OTSM) could contribute to the Engineering as Collaborative Negotiation (ECN) process instrumentation and evolution. First, decision-making and optimisation approach are compared with OTSM-TRIZ problem stating approach and overall strategies to perform steps of the ECN process are deduced. Then, it is briefly shown how OTSM-TRIZ deals with exploration and construction concepts that should both take place in ECN process. Finally, a general description of the Problem Flow Networks (PFN) approach based on OTSM-TRIZ technologies is given to show how it could be used within ECN process.
\end{abstract}

Keywords: TRIZ; theory of inventive problem solving; OTSM; ECN; engineering as collaborative negotiation.

Reference to this paper should be made as follows: Khomenko, N., De Guio, R. and Cavallucci, D. (2009) 'Enhancing ECN's abilities to address inventive strategies using OTSM-TRIZ', Int. J. Collaborative Engineering, Vol. 1, Nos. 1/2, pp.98-113.

Biographical notes: Nikolai Khomenko is currently Professor and Researcher at INSA Strasbourg Graduate School of Science and Technology. He is also member of research group of Innovative Process at European Institute of Energy Research (EIfER, Karlsruhe, Germany), and Leader of international educational project - Jonathan Livingston. In cooperation with the JL-project, educational programme Advanced Master of Innovative Design was developed as well as some other educational programmes in Europe, Asia and North America. He is also Leader of Minsk TRIZ-school since 1985. To disseminate results of his research and other OTSM-TRIZ knowledge. He was also invited by various organisations to give lectures around the World on TRIZ and OTSM and is author of many papers and two books published in 2003 and 2005.

Roland De Guio is Full Professor of Industrial and Production Engineering at INSA Strasbourg Graduate School of Science and Technology. His current research interests are the problems to tackle to facilitate the use of inventive problem-solving approaches in engineering and organisational design.

Denis Cavallucci is an Associate Professor at INSA Strasbourg Graduate School of Science and Technology where he teaches inventive design and is 
responsible of INSA's Advanced Master in Innovative Design. He is the Founder of TRIZ France association and actual President of European Triz Association (ETRIA); his research interests are oriented towards inventive practices in Engineering Design. He has given lectures worldwide on TRIZ and is regularly publishing the results of his research in international journals and scientific conferences. He also wrote chapters about TRIZ in co-authored books about the Science of Engineering Design. He is also active in establishing research cooperations with industrial organisations and is co-inventor of about 20 patents.

\section{Introduction}

As it has been defined in Lu and Jin (2005), Engineering as Collaboration Negotiation $(\mathrm{ECN})$ can be understood as a socio-technical decision-making activity one among the grounding hypotheses of which states that actors carrying different expertise and mixed motives are aiming at resolving conflicts and co-constructing 'consensual agreements' related to their common projects ( $\mathrm{Lu}$ et al., 2000). The expression "consensual agreement" is also defined as "good enough to meet the interests of all involved stakeholders". More precisely, ECN process consists of the following eight iterative steps (Lu and Jin, 2005):

1 "Individual analysis: Each party thinks alone to decide their respective Best Alternative To a Negotiated Agreement (BATNA)

2 Communal analysis: Two parties get together to establish their Initial Zone of Possible Agreements (IZOPA)

3 Mutual exploration: Both parties jointly explore maximal technical feasibilities

4 Establish preference: Parties jointly and collaboratively establish a value structure

5 Initial agreement: Parties locate initial agreements along the efficient frontier within the negotiation feasibility region

6 Joint co-construction: Parties $^{1}$ work together to dynamically and collaboratively modify their previously constructed value structure to improve initial agreements

7 Collective innovation: Parties collaboratively probe each other's knowledge to expand, or invent, new technical feasibilities for even more improved agreements

8 Collaborative negotiation: Parties simultaneously perform Steps 6 and 7, which is the ultimate goal of ECN."

Theory of Inventive Problem Solving (TRIZ) is an inventive problem-solving theory developed by G. Altshuller. History of TRIZ has already been expressed (Altshuller, 1986). Altshuller investigated thousands of patents, history of technological system evolution and most published known scientific discoveries appearing in encyclopaedia and collected state of the art publications. The result of this research was a set of methods and tools designed as a system to solve real and non-typical problems. Three axioms constitute the scientific background of classical TRIZ (Cavallucci et al., 2005). In addition to these three axioms, a model of problem-solving process was proposed. 
Altshuller named it ARIZ and this theoretical model was constantly developed and improved until 1985 as deductive unification of the theory occurs (Figure 1). The reader could refer to Altshuller $(1969,1975,1999)$ and Altshuller and Filkovsky (1976) to get more details about ARIZ.

Figure 1 Evolution of TRIZ's body of knowledge (see online version for colours)



As for any research work, limitations and direction of improvement of TRIZ have been established upon conclusions regarding many practical situations:

- Deductive unification allows a theory to develop but often suffers from a lack of axiomatic reformulation. This gives rise for new research in the clarification of TRIZ body of knowledge both in terms of semantic description and representation formalism.

- $\quad$ TRIZ addresses initial situations that can be stated with one or a few conflicting pairs of opposite technical contradictions, but the necessity to analyse initial situations that lead to deal with hundreds of contradictions appeared.

- Once general description of a solution is obtained, it has to be transformed into solution relevant to the specific situation. Altshuller's ARIZ also contains stages for preliminary evaluation and further development of a solution as well as a stage dedicated to reflection about specific problem-solving process and further improvement of ARIZ itself, but those stages are lagged in their evolution compared with stages of problem analysis and contradiction resolution.

- Since TRIZ possesses a semantic built with engineering grounding elements, its usage was more appropriate for engineering problem. But, in the 1980s appeared the need to deal simultaneously with engineering and non-engineering problems. As dialectical analysis seems to be a universal concept (Brachman et al., 1990), attempts to generalise TRIZ approach to non-engineering areas were made, and limitations were pointed out. To improve ability of TRIZ to solve complex cross-disciplinary problem, Altshuller proposed to transform Classical TRIZ into a general approach of problem solving; he proposed to name it "General Theory of Powerful Thinking", the Russian acronym of which is OTSM (Khomenko and Kucharavy, 2002). 
These limitations gave rise to research in the field of inventive problem solving and in the mid-1980s; some research directions were initiated under the acronym OTSM. Today, OTSM is still under development, but some tools to address previous stated limitations, which consist in four main technologies, were developed:

- new problem technology

- typical solution technology

- contradiction technology

- problem flow technology.

These technologies are to be harmonised into a meta-method named "Problem Flow Networks (PFN) Approach", the function of which is similar to ARIZ in classical TRIZ. TRIZ technologies are integrated in OTSM technologies and some results out of OTSM can be used to enhance TRIZ but they are not consensually agreed in TRIZ community. In this paper, the term 'classical TRIZ' refers to the consensual work of Altshuller, whereas the term OTSM-TRIZ refers to OTSM that includes TRIZ technologies improved by findings made during OTSM development.

Section 2 of this paper compares decision-making and optimisation approach, on the one hand, with OTSM-TRIZ problem-stating approach, on the other hand. Overall strategies to perform steps 3 and 7 of the ECN process are deduced from this comparison. Section 3 briefly shows how TRIZ deals with the exploration and construction concepts that also takes place in ECN process. Section 4 of the paper gives a general description of the PFN approach of OTSM-TRIZ to show how it could be used within ECN process.

\section{OTSM-TRIZ, optimisation, decision and innovation}

The space of search of ECN decision-making process may evolve regularly through an innovation process. The overall optimisation and decision-making process are well formalised. We shall show that using that OTSM-TRIZ solving problem can be described using the same elements and compared. Conclusions about the combined used of these two approaches within ECN process are proposed.

We shall consider successively a set of design problems, the requirements of which concern two evaluation parameters denoted EPI and EPII. A point in Figure 2 represents a solution of these problems. These solutions are mapped with a set of technical alternatives, the elements of which are known by the engineers. We assume that the set of technical alternatives includes all the typical solutions to this kind of problem. These solutions are described by a set of design parameters. Evaluation parameters are functions of design parameters. Let us call EPI-dp and EPII-dp the set of parameters that influence the values of EPI and EPII, respectively. EPI-dp and EPII-dp are defined by the set of technical alternatives.

In the first situation, the requirement targets EPI and EPII values to be within the range [EPI-1, EPI-2], [EPII-1, EPII-2], respectively. Thus, a solution fits the objectives when EPI and EPII define a point inside the rectangle ABCD in Figure 2. We also assume that known technical solution allows getting separately among targeted values of EPI and EPII. Thus, when there are no common design parameters between the 
evaluation parameters (i.e., EPI-dp $\cap$ EPII-dp $=\varnothing$ ), they are independent, and there is no problem to reach any point in the rectangle $A B C D$. But, when at least one design parameter influences both evaluation parameters EPI and EPII, they are dependent. This dependence relation constraints the area of feasible solutions in the evaluation space; it is represented by a curve in Figure 2. When the situation of the relation between the parameters overlaps the requirements area like in Figure 2, finding a solution can be considered as an optimisation problem. On this example, the common parameter is called $\mathrm{Px}$ and each value V of Px defines a point of the curve. The problem is then to find the values of Px that allows the evaluation parameters EPI and EPII to fit jointly the requirements. We can then enter a decision process by adding preferences about the pairs of evaluation parameters.

Figure 2 Optimisation situation (see online version for colours)



Let us now consider a second situation as summarised in Figure 3: the only difference with the previous situation is that the targeted area for the evaluation parameters is not overlapping the area of possible solutions defined by the design parameters. The relation between the evaluation parameters owing to the technical known solutions and natural law or laws that drive links between parameters remains the same. Thus, there is no way to get a solution by using this model of relation between the evaluation parameters, and a new model is required in which the relation between them overlaps the requirements. Two main approaches, which can be performed in synergy, may be used to perform this overlapping. The first attitude consists in changing our values about the preferences and keeping both the set of technical alternatives and the structure of the system. The second method consist in changing the set of technical alternatives and the structure of the system by expanding knowledge and inventing what is called in TRIZ language non-typical solutions. When applying the second method, we are dealing with an innovation situation OTSM-TRIZ contributes to implement the second approach by guiding the process for producing technical alternatives and structure of the system that fit the evaluation requirements. We shall now explain how OTSM-TRIZ suggests going further from this point using preferences to define ideality and stating the initial problem through a system of contradictions that will allow constructing appropriate new technical alternatives and structure of the system. As an output of this process, new curves between the evaluation parameters are generated. If they overlap the preference area, we come back to an optimisation situation. 
Figure 3 Innovation situation (see online version for colours)

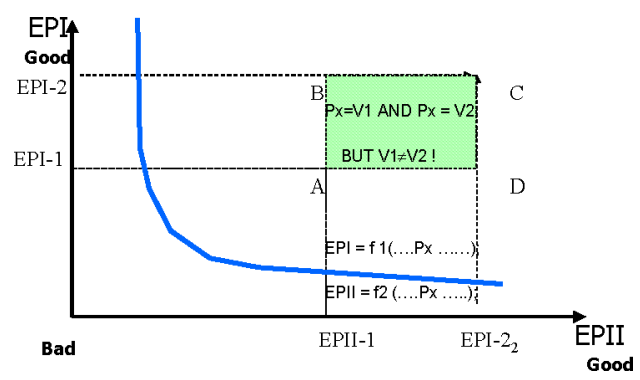

\subsection{Getting the first contradiction model}

The previous examples concerning the relation between evaluation parameters can be generalised and stated in the following way: the fact that two evaluation parameters are linked means that at least one common parameter of both of them depend on does exist (Sujan et al., 2002). These common parameters have to be disclosed to develop new technical alternatives and finally new structure of the system. Thus, in our example the fact that EPI and EPII are linked means that at least one common parameter Px the evaluation parameters (EPI and EPII) depend on does exist. The reason why it is impossible for the evaluation parameters to fit the requirements in the framework of existing models is the following: to fit jointly a pair (EPI, EPII) of the evaluation requirements, Px should take two mutually exclusive values; let us call them V1 and V2. Moreover, once taking into account partial elements of the preference structure, the situation can be described at least by three dilemmas. ${ }^{2}$ Let us illustrate this point through the previous example. We assume that the preference elements are:

- in the range [EPI-1, EPI-2], the higher EPI value the better

- $\quad$ in the range [EPII-1, EPII-2] the higher EPII value the better.

The three resulting dilemmas TC1, TC2 and PC become:

TC1: When EPII value is good from the requirement's point of view, then EPI is bad

TC2: When EPI is good from the requirement's points of view, then EPII is bad

$P C$ : When Px value equals V1, then TC1 holds whereas when Px value is V2 then TC2 holds.

The PC dilemma concerns a choice between two mutual exclusive values of a parameter that leads to two options TC1 and TC2 that are unfavourable from the requirement's points of view. The link between the above mentionned dilemmas is summarised in Figure 4.

OTSM-TRIZ does not take as starting point, the decision-makers' point of view that deals with dilemmas, but the point of view of the problem-solver that targets to reach a goal composed of conflicting and contradictory requirements in existing models of the structure of the system. The designer has to overcome the discrepancy between desired result and the results that can be obtained through the existing models. Let us note that the systematic and explicit connexion between PC, on the one hand, and TC1 and TC2, on the other hand, are not developed in classical TRIZ but are part of OTSM-TRIZ 
approach. In classical TRIZ, TC1 and TC2 are named technical contradictions whereas the underlying contradiction PC corresponds to the concept of physical contradiction. In OTSM-TRIZ, these two concepts are generalised; $\mathrm{TC} 1$ and $\mathrm{TC} 2$ are called Contradictions of a System - CS - (conflict between two different parameters of the same system) whereas PC is named Contradiction of a Parameter - CP - (conflict between two different values of a same parameter). We present here just two types of contradictions: CS and CP. Complete OTSM system of contradictions discloses deep roots of the problem and proposes ways either to eliminate the roots of the problem themselves or take them into account and bypass them along problem-solving process. Classical TRIZ system of contradictions has just three types of contradictions (administrative, technical and physical) whereas OTSM-TRIZ typology of contradictions is more complicated to describe problem-solving process a more formal way and more precisely.

Figure 4 OTSM-TRIZ basics system of contradictions



OTSM-TRIZ contains a technology called 'new problem technology' for seeking the set of contradictions to be solved to reach requirements. This new model of problem is the standard starting point for the next steps towards a new model that fits the requirements using other OTSM-TRIZ technologies in frame of PFN approach.

\subsection{Ideality and preferences}

The imaginary point of the preference space the coordinates of which are defined by the most desirable value of each evaluation parameter is called ideal point. TRIZ strategy aims at building solutions getting as close as possible of this ideal point. The ideal point becomes the target in OTSM-TRIZ approaches. Let us remark that in the frame of OTSM-TRIZ, a concept of ideal solution is developed that is very similar to ideal point but there remains differences.

\subsection{Optimisation vs. innovation in ECN process}

We shall now analyse the two previous examples in the frame an ECN process. It will lead us to discuss steps 3 and 7 of ECN process. We assume in this part of the discussion that we have at our disposal, on the one hand, a preference structure and that we can define an imaginary ideal or most desirable point and, on the other hand, an approach that allows to get as close as possible to this ideal point through an innovation process. 
To analyse the first initial situation with the eyes of an ECN approach, we should reformulate it. The situation is summarised in Figure 5. Let us assume that there are two negotiators I and II. The parameters EPI and EPII are the evaluation criteria for negotiators I and II, respectively. The most desirable values for negotiators I and II are EPI-2 and EPII-2, respectively (BATNA). The acceptable individual alternatives for negotiators I and II are within the range [EPI-1, EPI-2] and [EPII-1, EPII-2], respectively. The aggregation of these individual points of views leads to a common Initial Zone of Possible Agreements (IZOPA) that is represented in Figure 5 by the area inside the rectangle $\mathrm{ABCD}$. The curve that overlaps this area represents the evaluation parameters of actual available technologies. Thus, point BI and BII are the points associated to the best solution for negotiators I and II, respectively. The part of the curve between BI and BII represents the Zone of Possible Agreements (ZOPA) as defined in the ECN model given in the introduction of the paper. The negotiation will lead to a consensual agreement inside the ZOPA; let us call it $\mathrm{Ca}$. The zone in the hatched zone of the IZOPA is the set of imaginary points that dominate $\mathrm{Ca}$. From this stage, a point in the value space will represent any innovation activity that lead to a new feasible technology. The new solutions produced by this innovation process will only be valuable from the negotiation point of view if their evaluation parameters belong to the area of the IZOPA that dominates Ca. OTSM-TRIZ technologies contain rules that guide and facilitate the process for building innovations whose evaluation parameters belong to the area that dominates Ca. Thus, these technologies can be used in step 7 of the ECN process for building a win-win solution instead of a trade-off.

Figure 5 Optimisation or innovation? (see online version for colours)

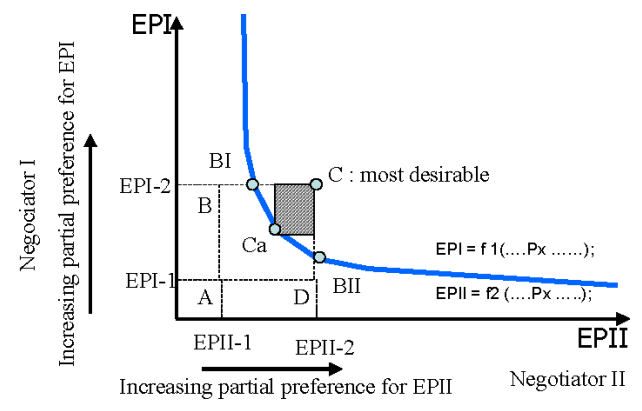

The second initial situation can be reformulated in the ECN context using the same notations than for the first initial situation; it is summarised in Figure 6. Step 3 of ECN process (Mutual exploration) did not bring any feasible technical solution, the evaluation parameters of which belong to the IZOPA. Thus, negotiation could lead the negotiators to either modify their initial IZOPA to make the new ZOPA fit the curve of feasible technical solutions and get into an optimisation or decision process, or enter into an innovation process that intends to produce a solution in the IZOPA. Again, OTSM-TRIZ could be used to perform this innovation stage.

The two previous examples suggest us to propose as requirements of step 7 of the ECN process that are given in a decreasing order of priority: 
1 Step 7 activities have to be oriented to the most desirable solution

2 Step 7 activities have to be oriented to the ZOPA

3 Step 7 activities have to be oriented to build solutions that dominate consensual agreements.

Figure 6 Typical situation for potential innovation (see online version for colours)

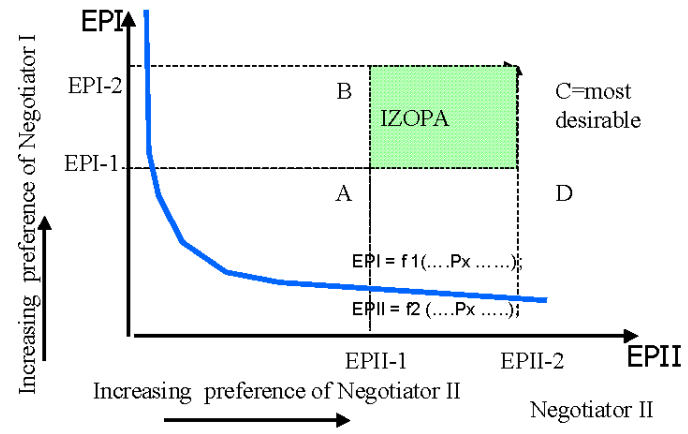

A first consequence of the acceptance of previous requirements on the tooling of the step 7 activities can be stated in the following way: as the knowledge expansion and technical innovations are goal oriented, the tools should allow minimising short-term and long-term innovation costs. Research has to be performed in this direction.

A second consequence of the acceptance of the previous requirements concerns the order of steps 3 (Mutual exploration) and 4 (Establish preferences) in the ECN process. Indeed, one may pose the question: to what extent is it interesting to get all the solutions that are out of the IZOPA? If preferences (step 4) are established after step 2, step 3 can become a mutual exploration guided by the preferences, thus a method to minimise exploration costs can be designed.

Today's optimisation approaches deal with exploration of the existing solutions while innovation deals with enlarging the set of possible solutions. The way to combine them efficiently within ECN process remains an open question; nevertheless, hints can be extracted from OTSM-TRIZ methodology. It is the purpose of the next section.

\section{Exploration vs. innovation}

In this section, we shall focus on two main problem-solving approaches namely searching and constructing solutions. Experience about how OTSM-TRIZ deals with them within the innovation process could help ECN development. In the framework of inventive problem solving, search for solutions is the more popular but the less efficient approach when addressing difficult and very new problem solving; we shall show its limitations. Search presupposes the existence of a ready-to-use solution while construction approach presupposes the existence of know-how that allows constructing a solution. OTSM-TRIZ was designed to implement the second approach while using exploration approach as well. 


\subsection{Exploration: searching for solutions}

The notion of search is often associated to the idea of trials, which may be eventually fruitless. It may also be linked to more positive ideas like exploration of information (Sowa, 1994), curiosity and discovery. These concepts express feelings linked to search activity but do not specify the search activity. In the framework of invention, effectiveness and efficiency are often considered as diametrically opposed to searching and a finding is often associated to chance. A widespread approach to exploration is a succession of more or less efficient trials and errors. The simplest version of this approach consists in trying successively all the operational solutions that were capitalised by different means and evaluating them. This approach is effective when operational solutions do already exist and when they are quickly accessible. Thus, this approach is suitable for limited area of knowledge. When the domain of knowledge required for solving the problem is too large for applying previous search approach efficiently, problem can be solved using analogy (Minsky, 1975). Various other ways can be used to improve exploration speed. Operational research provides efficient ways to perform exploration as well as limits of this approach. As a concluding remark of this section, let us notice that efficient search is linked to the following two conditions:

- Pre-existence of the solution.

- Availability of means that quickly allow finding the pre-existing solution.

A corollary of this condition is that in case no solution pre-exists, means to detect this situation quickly should also be available.

In the body of knowledge of Classical TRIZ, it was established that five levels of inventiveness characterised the inventions presented in the studied patents (Altshuller, 1984). Level 1 represents a corpus of patent with non-solved contradictions thus this level has been qualified as 'trivial solutions'. The second level start to present a solution to a contradiction (showing a non-compromise attitude) but its level has been stated as 'minor improvement' because the investigated scope of knowledge did not exceed the one in which the problem was posed. Then, the third level was defined as 'major improvement', whose peculiarities was not only to solve a contradiction, but also to solve it in using elements extracted from knowledge found in technological fields outside the one where the problem was posed. The fourth level characterised the 'breakthrough' solutions, where the solution to a contradiction was collected from fundamental sciences (physics, chemistry or geometry, etc.) and thus the peculiarity has not been applied yet in industrial productions (produced massively) in any fields of engineering but to have been scientifically proved in research laboratories. The fifth and last level concerns mostly the inventions showing a total new knowledge creation, mostly present in discoveries, these solutions characterise the pioneer patents and have the peculiarities to subsequently generate new generations of patents of a lower inventiveness level based on the same novelty.

From these studies, we have learned an important notion in inventive problem-solving framework: Assuming that a solution in a specific field of knowledge becomes a standard solution, one of the major challenges was to organise an appropriate mapping of other fields of knowledge to move from non-standard solution to standard solution of the same problem. Thus, reformulating a problem with the aim to lower the level of inventiveness can become a challenge in inventive problem solving. As an example, we would like to 
use the solution actually in use in plastic injection mould design to manufacture internal walls of cavities of a mould to obtain a 'skin-like' surface of a plastic part. Several years ago, it was proposed to use conventional machining machines to treat the internal surface of cavities to reproduce the proper surface effect. These solutions were not satisfying regarding the quality and time spent to obtain a natural skin effect until the problem was transposed in another field of knowledge (chemistry) where the solution to use oxide-reduction to attack the steel surface in a random way. The problem seemed to be unsolvable (non-standard) in mould-making industry until it was reformulated as chemistry problem, then a trivial (standard) solution for domain of chemistry was found.

The first steps of Classical-TRIZ evolution were oriented to the organisation of knowledge to built efficient search approach. Next steps of TRIZ evolution concern construction of pretty new solutions.

\subsection{Building a solution}

In certain situations, the use of several standards, inventive principles and even non-catalogued concepts in Classical TRIZ knowledge bases ${ }^{3}$ are required. OTSM-TRIZ proposes methods that facilitate effective combinations of concept that are not solutions of the problem but that contain ideas that partially solve the problem; these concepts are called partial solutions in OTSM-TRIZ language. A partial solution would be rejected as solution of the problem because its implementation may gender undesirable or negative effects, which, sometimes, dominates its positive effects. This is the reason why partial solutions are useless in exploration strategies mentioned earlier, whereas they are as much bricks that will help to built the future solution within construction strategies. Partial solutions are identified along the OTSM problem-solving process based on various kinds of standard and other typical solutions of OTSM-TRIZ. Along this process, the solver aggregates partial solutions into Converged Conceptual Solutions by the means of OTSM-TRIZ knowledge, his or her personnel knowledge and skills of specialists of various but targeted domains. Convergence of partial solutions is performed to increase systematically positive effects while decreasing systematically eventual negative effects. When positive effects surpass enough the negative one, the convergent concept becomes the Final Conceptual Solution; otherwise, it is considered as a new partial solution and generation of partial solutions continue in respect of OTSM-TRIZ rules and mechanisms.

Just as search mechanisms, there are different ways to get construction mechanisms. The challenge is to build effective and efficient ones; it is the goal of the PFN Approach.

\section{Problem flow networks approach and negotiation process}

This section aims at answering the question: "how could PFN approach help negotiation process?" Before discussing this question, basic information about PFN approach are given. To explain some key points of this approach, the main tool of Classical TRIZ, Altshuller's ARIZ, is briefly described.

\subsection{A brief sketch of ARIZ}

At the beginning, the Initial Problem situation is analysed and underlying Technical Contradictions should be discovered. A Technical Contradiction in classical TRIZ is a 
contradiction between two evaluation parameters of a system as was described earlier. The first part of ARIZ process leads from initial problem situation description to a model of the problem described as a technical contradiction, which is based on the initial technical contradictions discovered.

The next stage aims at analysing the model of problem obtained at previous stage. Along this stage, resources of problem situation that may be used to solve the problem are analysed, namely space, time, substances and field resources, when dealing with engineering domain. The result is used afterwards to discover the roots of the Technical Contradiction: the Physical Contradictions relevant to substance and field resources discovered before. A Physical Contradiction is a contradiction between two opposite or incompatible values of a same design parameter. Classical TRIZ typical tools can then resolve the Physical Contradiction.

As we can see, ARIZ problem-solving process for non-typical problems finally leads to a problem description that is typical for Classical TRIZ. In other words, we could say that problem that initially looks like not typical along the classical TRIZ problem-solving process is progressively transformed into a typical problem that can be solved by TRIZ typical solutions. The reader who is familiar with ARIZ will find the previous description of Classical TRIZ problem-solving process very simplified but it seems enough in the scope of our present talk. The way ARIZ works that is described earlier was the mainstream of the almost 40 years of evolution of ARIZ (1946-1985). But, in Altshuller's last versions, which were developed in the middle of 1980s, appear new interesting ideas that evolved during the last 20 years into the PFN approach.

\subsection{Problem Flow Network approach}

Problem often seems difficult because many elements and their parameters influence each other. Classical TRIZ postulates that the difficulty is due to an underlying contradiction between linked parameters. The contradiction model is also a very powerful model to understand the root of problems (Sujan et al., 2002). Complex problem situations are characterised by much large set of parameters and interference of elements involved into the problem. For instance, many Physical Contradictions may be linked to a given pair of Technical Contradictions. The more numerous and complicated these interconnections the more difficult the application of classical TRIZ tools.

General outline of PFN approach is similar to the one of the ARIZ in classical TRIZ where a Network of Problem, a Network of Contradictions and a Network of Parameters replace the Initial Problem situation, the Technical Contradiction and the Physical Contradiction, respectively. In addition, PFN approach brings more precise instruments than classical TRIZ to understand and describe problem situation in problem-solving context. Another difference with classical TRIZ is the opportunity provided by PFN to evaluate and compare solutions more objectively with the knowledge that was disclosed along OTSM-TRIZ problem-solving process. And finally, PFN provides solvers with a tool, which is almost absent in ARIZ, for adjusting a general typical solution, which is usually provided by ARIZ, to a specific situation.

For certain specific cases, we could go further and transform the specific Networks of Parameters into a general Network of Parameters that holds for a larger domain of knowledge. This general knowledge can then be memorised for reuse in future problems. It looks like ECN approach that could be one of the cases to apply General Network of 
Parameters as well. Some other points of PFN approach interesting for ECN applications are given below.

\subsection{Construction of a network of problems}

Depending on the initial situation and the level of OTSM-TRIZ education of the team members that address the problem, there are several options to start the construction of the Network of Problem. Most common situation is the one where people do not know so much about neither classical TRIZ nor OTSM-TRIZ. In this case, a usual starting point is a list of problems, difficulties, inconveniences and questions relevant to the problem situation made by professionals dealing with the problem. Then, along a negotiation process using OTSM-TRIZ rules and technologies, this initial list of problems is transformed into a Network of Problem. This network can be considered as a semantic network or as a graph; it could be presented graphically or in the shape of a connectivity matrix. Both ways of graph representation are used to analyse the Network of Problems. The nodes of the graph represent the problems whereas the edges represent the link between the problems. Links between nodes of the graph can be interpreted as relations between problems (nodes), thus the graph is oriented. Orientation of the links is done according to OTSM rules. The graph also contains nodes that present known Partial Solutions of certain problem nodes. The graph organisation produces a 'big picture' of the problem situation. Its analysis leads to disclose a set of key problems of the whole problematic situation. This analysis is also the result of negotiations about clarification of certain points of the initial problem situation.

Along the process of construction of the Network of Problems, the set of problem evolves. The available unsatisfying typical solutions can be used to generate new partial solutions for some of the problems, so they become sub-problems of given problems. All the OTSM-TRIZ approaches can be applied to these sub-problems. This property of OTSM-TRIZ problem-solving process allows getting a fractal model of the solving process. The problems can also be split or converged. Often new problems appear. Both known and new partial solutions appear as a node of the graph. Analysis of partial solutions and of their Positive and Negative points leads to some new problems that were not mentioned before or even were unknown for professionals of the domain. Once the Network of Problems becomes stable (does not evolve) and key problems are extracted, the set of Technical Contradictions that are behind the set of key problems can be disclosed and used as initial list of contradictions to construct the Network of Contradictions.

\subsection{From network of problems to the network of contradictions}

Network of problems is useful to understand problem and narrow the area of search for problem solving according to one of the main ideas of Classical TRIZ (Khomenko and Kucharavy, 2002) The Network of Problems gives a set of key problems of the whole problematic situation. To discover roots of these key problems, it is proposed to investigate them and represent them in the shape of Technical Contradictions according to rules of Altshuller's ARIZ and OTSM-TRIZ Contradiction Technology. The problems of the Network of Problems are also linked to the set of evaluation parameters of the system to be designed that should be improved according to the list of specifications. The Network of Contradictions contains the set of elements of the system and its 
environment, which are connected to the key problem discovered in Network of Problems that prevent from the improvement of Evaluation Parameters by typical well-known solutions. This network is analogue to Classical TRIZ model of problem presented in the shape of mono-contradiction. Nodes of the graph are parameters and Elements-components of the system or its environment relevant to the specific problem; the edge indicates which parameter influences positively or negatively the other parameter value in the context of the problem. Network of contradiction can be considered as a poly-contradiction. Next steps of OTSM problem-solving process are search about resources of the problem situation involved in the Network of Contradictions and transformation of the network of contradictions into the Network of Parameters according to rules of Altshuller's ARIZ and OTSM Contradiction Technology.

\subsection{The Network of Parameters}

The Network of Parameters is equivalent to the Physical Contradictions in ARIZ. It could be considered as a physical poly-contradiction or a network of physical contradictions. For engineering problems, this network gives a deep understanding of the discrepancy between the human wishes (target of the problem) and the natural laws of nature described in science (Kucharavy and De Guio, 2005). To solve the problems, we can resolve these contradictions by using Classical TRIZ and OTSM toolbox.

Above, we presented Network of Parameters for a specific situation. This network can be transformed into General Network of Parameters and used for future problem-solving sessions. General Network of Parameters is a set of objective laws of certain domain of knowledge that may more or less systematically generate specific contradictions of parameters when dealing with problems in this domain. The roots of the new specific problems are then discovered much faster in each specific situation. That is why this could be helpful for problem solving and solution evaluation as well.

\subsection{Objective evaluation and comparison of partial and Final Conceptual Solutions}

Along the PFN procedure, relevant information about the problem is made explicit and organised. This knowledge can be used to build criteria for comparing Final Conceptual Solutions or for evaluating Partial Conceptual Solutions along the problem-solving process. Network of Problems for instance can be useful to investigate whether all the problems of the network are solved and how efficiently they are solved. Furthermore, Networks of Parameters can be useful to forecast some consequences of the implementation of certain solutions.

\subsection{Application to ECN}

From OTSM-TRIZ point of view, negotiation process could be considered as a network of various problems that are linked together. This is a typical starting point to construct a Network of Problems. According to ECN assumption, which asserts that all negotiators are willing to reach common goals and getting mutually benefits of negotiation results, the Network of Problems seems to be an efficient way to provide guidelines for 
negotiations, helps to clarify their mutual interests and helps to clarify contradictions that prevent participants to satisfy those mutual interests.

As soon as ECN process of negotiations can be represented as a network of problems, this network could be converted into specific Network of Parameters relevant to a specific situation. As a result, it will help to disclose the roots of the problems and eventually redefine the system of goals of the negotiators. Then, contradictions can be solved using ECN and OTSM-TRIZ instruments. In case ECN sessions are happening regularly in the same organisation, it may be useful to turn it into a General Network of Parameters. It can then be reused in new specific situations and developed further during future ECN or problem-solving sessions.

\section{Conclusion}

ECN is a process that has to deal with present know-how and potential novelties. When good enough solutions do exist, decision-making model and search strategies are appropriate. Otherwise, an innovation strategy has to be undertaken. Getting effective and efficient innovation processes and tools that bring value to the stakeholders are issues. We showed that in the framework of ECN, innovation could be stated as a problem, the goal of which is to build a solution that dominates all existing solutions. To perform this, we propose to combine search technologies and construction technologies. We suggested ways to use OTSM-TRIZ to address these issues.

\section{References}

Altshuller, G.S. (1969) Algorithm of Invention, Moscowskiy Rabochy, Moscow, 1973.

Altshuller, G.S. (1975) Inventive Problem Solving Process: Fundamental Steps and Mechanisms, Manuscript, 6 April.

Altshuller, G.S. (1984) Creativity as an Exact Science: The Theory of the Solution of Inventive Problems, Translated by Anthony Williams, Gordon and Breach Science Publishers, ISBN 0-677-21230-5.

Altshuller, G.S. (1986) To Find an Idea: Introduction to the Theory of Inventive Problem Solving, Nauka, Novosibirsk, 1991.

Altshuller, G.S. (1999) The Innovation Algorithm: TRIZ, Systematic Innovation, and Technical Creativity, Technical Innovation Center, Worchester, Massachusetts, p.312, ISBN 0964074044.

Altshuller, G.S. and Filkovsky, G. (1976) Actual State of TRIZ, Baku, available in Russian at http://www.altshuller.ru/triz2.asp

Brachman, R.J., McGuinness, D.L., Patel-Schneider, P.F., Resnick, L.A. and Borgida, A. (1990) 'Living with classic: when and how to use a KL-ONE-like language', in Sowa, J. (Ed.): Formal Aspects of Semantic Networks, Morgan Kauffman, San Mateo, CA, pp.401-456.

Cavallucci, D., Khomenko, N. and Morel, C. (2005) 'Towards inventive design through management of contradictions', Proceedings of CIRP-2005 Conference, Shanghai, China.

Khomenko, N. and Kucharavy, D. (2002) 'OTSM-TRIZ problem solving process: solutions and their classification', Proceedings of TRIZ Future Conference, 6-8 November, Strasbourg, France.

Kucharavy, D. and De Guio, R. (2005) 'Problems of forecast', 5th TRIZ Future Conference, 16-18 November, Graz, Austria. 
Lu, S. and Jin, Y. (2005) Engineering as Collaborative Negotiation (ECN): A New Foundation for Collaborative Engineering Research and Practice, A Draft Working Whitepaper for the CIRP Working Group on 'Engineering as Collaborative Negotiation' (WG-ECN), 15th International CIRP Design Seminar 2005, Shangai Jiao Tong University, Shangai, China, p.28.

Lu, S., Cai, J.C., Burkett, W. and Udwadia, F. (2000) 'A methodology for collaborative design process and conflict analysis', CIRP Annals, Vol. 49, No. 1, pp.561-566.

Minsky, M. (1975) 'A framework for representing knowledge', in Winston, P. (Ed.): The Psychology of Computer Vision, McGraw-Hill, New York.

Sowa, J.F. (1994) Conceptual Structures: Information Processing in Mind and Machine, Addison-Wesley, Reading, MA.

Sujan, M., Rizzo, A. and Pasquini, A. (2002) 'Contradictions and critical issues during system evolution', Proceedings of the ACM Symposium on Applied Computing, Madrid, Spain, ISBN 1-58113-445-2.

\section{Notes}

${ }^{1}$ As TRIZ is a set of method organised as a system it fits one of the definitions of the word theory.

${ }^{2}$ Dilemma is understood here as a situation that requires a choice between options that are or seem equally unfavourable or mutually exclusive.

${ }^{3}$ The fact that a concept is not catalogued does not systematically imply that they are missing in TRIZ basic concepts; it may be an aggregation of basic concepts. 